\title{
Systemic Therapy of Bronchioloalveolar Carcinoma: Results of the First IASLC/ASCO Consensus Conference on Bronchioloalveolar Carcinoma
}

\author{
Mark G. Kris, MD, * Giuseppe Giaccone, MD, † Angela Davies, MD, $\neq$ Masahiro Fukuoka, MD,, \\ David H. Garfield, MD,|| Jacek Jassem, MD, 9 Elisabeth A. Quoix, MD, \# Alan B. Sandler, MD, ** \\ Giorgio V. Scagliotti, MD, †† Jan P. Van Meerbeeck, MD, PhD, t+ and Howard West, MD $\S$
}

Introduction: Bronchioloalveolar carcinoma (BAC) is a subtype of adenocarcinoma of the lung with unique pathological, clinical, and molecular characteristics.

Methods: This consensus conference group reviewed studies performed specifically in BAC and data from patients with BAC who were included in clinical trials of all non-small-cell lung cancer (NSCLC) subtypes.

Results: Although BAC as defined by the World Health Organization represents less than $5 \%$ of adenocarcinomas, as many as $20 \%$ of adenocarcinomas have BAC features. These latter tumors are more likely to have mutations in the epidermal growth factor receptor (EGFR) gene and to be sensitive to the EGFR tyrosine kinase inhibitors gefitinib and erlotinib. Although most patients are men and have a history of smoking cigarettes, proportionally more are women and never smokers. Patients with BAC are routinely treated with drugs and regimens appropriate for patients with all subtypes of adenocarcinoma of the lung; four studies have been performed specifically in this disease.

Conclusions: There is insufficient evidence to confirm or refute the assertion that the sensitivity of BAC to chemotherapy is different from that of other lung cancer histologic types. The unique clinical and molecular characteristics associated with BAC led this panel to conclude that future clinical trials should be designed specifically for persons with BAC. Recommendations for trial design and research questions are proposed.

From the *Memorial Sloan-Kettering Cancer Center, New York, NY; †Free University Hospital, Dept of Oncology, Amsterdam, The Netherlands; $\$$ University of California Davis, Division of Hematology/Oncology, Sacramento, CA; §Iinki University School of Medicine, Dept of Medical Oncology, Osaka, Japan; |University of Colorado Comprehensive Cancer Center, Denver, $\mathrm{CO} ; \boldsymbol{\uparrow M e d i c a l}$ University of Gdansk, Oncology and Radiotherapy, Gdansk, Poland; \#Service de Pneumologie Lyautey, Strasbourg, France; **Vanderbilt University Medical Center, Nashville, TN; $\dagger \dagger$ University of Torino, Dept Clinical and Biological Sciences, S. Luigi Hospital, Orbassano, Italy; \$tUniversity Hospital, Respiratory Diseases, Ghent, Belgium; and the $\S \S$ Swedish Cancer Institute, Medical Oncology, Seattle, WA.

Address for correspondence: Mark G. Kris, MD, Memorial Sloan-Kettering Cancer Center, 1275 York Avenue, New York, NY 10021. E-mail: krism@mskcc.org

Copyright (C) 2006 by the International Association for the Study of Lung Cancer

ISSN: $1556-0864 / 06 / 0109-0032$
Key Words: Adenocarcinoma, Bronchioloalveolar carcinoma, Epidermal growth factor receptor, Gefitinib, Erlotinib.

(J Thorac Oncol. 2006;1: S32-S36)

B ronchioloalveolar carcinoma (BAC) may be distinguished from other adenocarcinomas of the lung by unique histology, radiology, molecular biology, and clinical course. It is thought that the course of patients with BAC is more indolent than in persons with other non-small-cell lung cancer (NSCLC) histologies. Although "pure" BAC is defined explicitly by the World Health Organization (WHO), ${ }^{1}$ there are many more patients whose adenocarcinomas have features of $\mathrm{BAC}$, making the histologic classification of such tumors uncertain. $^{2}$ Several clinical trials have been performed specifically in patients with BAC, based on the notion that these tumors may be somewhat less sensitive to standard chemotherapy and that the biology of BAC differs from that of other adenocarcinomas.

\section{MATERIALS AND METHODS}

This conference group reviewed results of patients with BAC included in trials studying patients with NSCLC in general and five studies designed specifically for persons with BAC, testing paclitaxel, gefitinib, and erlotinib. Local treatment approaches are also outlined here, including inhalational therapy with investigational anticancer agents. The conference group made recommendations for clinical trial design and further research in BAC.

\section{RESULTS}

\section{Results in Patients with BAC Included in ECOG Trial 1594 in Patients with Advanced NSCLC}

The Eastern Cooperative Oncology Group (ECOG) studied four platinum-based regimens as initial therapy for patients with NSCLC. ${ }^{3}$ This study included 1163 patients, 17 of whom were classified as having BAC by the investigator. The response rate in patients with BAC was $6 \%$ compared with $20 \%$ for patients with other types of NSCLC. Median 
survival for persons with BAC was 12 months compared with 8 months overall.

\section{Trials Conducted Specifically in Patients with BAC}

\section{Paclitaxel by 96-hour Infusion for Patients with BAC (SWOG9714)}

The Southwest Oncology Group (SWOG) conducted the first prospective trial for patients with BAC. ${ }^{4}$ Patients in this trial had ECOG performance status 0 to 2 and stage IIIB/IV BAC as determined by the treating institution. All 58 patients received paclitaxel $35 \mathrm{mg} / \mathrm{m}^{2} /$ day continuously infused for 96 hours every 21 days. The partial response rate was $14 \%$. Median overall survival was 12 months, and the 3 -year survival rate was $13 \%$. Because of a high treatmentrelated death rate $(9 \%, 5 / 58)$, this regimen was not pursued. However, this trial demonstrated the feasibility of conducting prospective trials in patients with $\mathrm{BAC}$ in a cooperative group setting and established benchmarks for future studies.

\section{Paclitaxel by 3-hour Infusion for Patients with BAC (EORTC 08956)}

The European Organization for Research and Treatment of Cancer Lung Cancer Group studied a 3-hour paclitaxel infusion in patients with stage IIIB/IV BAC. ${ }^{5}$ Nineteen patients received paclitaxel $200 \mathrm{mg} / \mathrm{m}^{2}$ every 21 days. Two partial responses were confirmed (response rate of $11 \%(95 \%$ CI, 1 to $35 \%$ ). The median survival was 9 months, and the 1 -year survival rate was $35 \%$ (95\% CI, 14 to $56 \%$ ). The study was terminated because of a low response rate.

\section{Gefitinib (Iressa) for Patients with BAC (SWOG 0126)}

SWOG S0126 evaluated $500 \mathrm{mg}$ of gefitinib per day. ${ }^{6}$ The study was undertaken based on the detection of epidermal growth factor receptor (EGFR) protein in BAC tumors collected from S9714, the activity seen in patients with NSCLC in phase I trials of gefitinib, and reports of rapid and durable responses in patients with BAC..$^{7,8}$ This study enrolled 55 patients with eligibility identical to the preceding S9714 trial along with 35 previously treated patients. All had performance status of 0 to 2 and stage IIIB/IV BAC as determined by the treating institution. Approximately one third of patients had a dose reduction to $250 \mathrm{mg}$ based on toxicities, and $11 \%$ discontinued therapy. Three patients developed increasing pulmonary infiltrates and hypoxemia requiring treatment with steroids, antibiotics, and supplemental oxygen after discontinuing gefitinib. Each of these patients died of what was possibly treatment-related interstitial lung disease, although disease progression, infection, or other causes may have contributed. Among patients with measurable disease, gefitinib response rates (RECIST) were 19 and $9 \%$ for chemo-naïve and chemo-pretreated patients, respectively. Complete responses were seen. The median survival of untreated patients was 12 months, similar to that in SWOG 9714. Several patients had progression-free survival of more than a year, something not observed with paclitaxel. The survival at 2 and 3 years was 38 and 23\%, an improvement on the 29 and 13\% seen in S9714. Patients with nonmucinous BAC had superior survival on gefitinib compared with those with the same histologic features treated with paclitaxel on protocol S9714 discussed above. ${ }^{9}$ Increased EGFR gene copy number detected by fluorescence in situ hybridization was associated with improved survival. ${ }^{10}$ Correlations of clinical outcomes with EGFR protein expression measured by immunohistochemistry and EGFR mutations are ongoing.

\section{Erlotinib (Tarceva) for Patients with BAC}

During the early testing of erlotinib, dramatic responses were observed in patients with BAC. To follow up on this lead, four centers undertook a phase II trial of erlotinib 150 $\mathrm{mg}$ daily in patients with BAC - both "pure BAC" as defined by the WHO and adenocarcinoma with BAC features. ${ }^{2,11} \mathrm{~A}$ total of 159 patients with stage IIIB or IV inoperable NSCLC had central review of biopsy specimens. BAC was identified in $66 \%$ of submitted specimens. Like the SWOG studies, pathological eligibility was determined by the reading from the pathology department where the patient was treated, not by central review. One hundred one patients were treated; $67 \%$ of the patients were women, and $22 \%$ had previously received chemotherapy. Central pathology review showed that $12 \%$ were "pure BAC" and that $88 \%$ had adenocarcinoma with BAC features. Twenty-five percent of the patients had never been smokers. A final report is planned for the first half of 2006.

Erlotinib treatment led to partial responses (RECIST criteria) in 20 of the 84 patients evaluated at the time of the report (24\%; $95 \%$ CI, 16 to $34 \%$ ). The partial response rate was $40 \%$ in never smokers and $19 \%$ in current or former smokers. The 1-year survival rate rfor all patients was 58\%; the median survival rate has not yet been determined. There was one case of interstitial lung disease resulting in treatment-related death. EGFR exons 18 to 24 have been sequenced in 19 tumor specimens to date. Among nine patients with partial responses, six had EGFR mutations, two with deletions in exon 19, and four had substitution mutations in exon 21 (L858R). ${ }^{12}$ None of the 10 patients who did not have partial responses had a mutation in EGFR exons 18 to 24 . The trial closed to accrual in April 2005. Studies of EGFR protein expression, EGFR copy number, sequencing of EGFR exons 19 and 21, and sequencing of KRAS exon 2 will be obtained from all patients with tumor tissue available. A final report is planned for the first half of 2006.

\section{Trial of gefitinib for patients with Pneumonic-Type Adenocarcinoma}

This prospective French Thoracic Oncology Intergroup 30-center phase II study (IFCT 04-01) opened in May 2004 to evaluate gefitinib $250 \mathrm{mg}$ daily as a first-line therapy for patients with nonresectable, pathologically proven, pneumonic-type adenocarcinoma as defined by Wislez et al. ${ }^{13}$ This group includes adenocarcinomas with both mixed-invasive and BAC-predominant subtypes. Interim results were reported at the International Association for the Study of Lung Cancer meeting in Barcelona, Spain in July 2005. ${ }^{14}$ Objective responses were documented in four of 24 patients reported 
(17\% observed rate, $95 \% \mathrm{CI}, 5$ to $37 \%$ ). Tissue will be analyzed for expression of EGFR, HER2, P-AKT, and MAPK as well as mutations in KRAS codon 12 and EGFR exons 18 to 21 . A final report on 90 patients is planned for the first half of 2006 .

\section{Other Strategies}

\section{Adenovirus P53 Administered by}

Bronchioloalveolar Lavage in Patients with BAC

Because BAC is characterized by thin sheets of tumor cells growing along the airways, agents delivered by inhalation should have direct access to the tumor. A phase I study of p53 gene transfer using an adenovirus vector (Ad-p53; RPR/INGN 201) delivered via bronchioloalveolar lavage (BAL) to involved lobes of the lung was conducted at the University of Wisconsin and Vanderbilt University. ${ }^{15}$ Treatments consisted of two BAL at the same dose level given 2 weeks apart to a single involved lobe. Additional treatment to all involved lobes was allowed if initial treatments were tolerated. The initial dose was $2 \times 10^{9}$ viral particles per dose, escalated in 10-fold increments. Among 14 patients registered, grade 4 pulmonary toxicity was noted at the $2 \times$ $10^{9}$ dose level. Two additional patients at this level did not suffer a dose-limiting toxicity. After the completion of two cycles, pathologic response was noted on rebiopsy in two patients, four of nine patients showed improved diffusing capacity, and four of 11 had symptomatic improvement.

\section{Autologous Antitumor Vaccine GVAX (CG8123)}

GVAX (CG8123) (Cell Genesys, Inc.) is a GM-CSF gene-modified autologous tumor cell vaccine that has shown antitumor activity in patients with NSCLC and, specifically, BAC. ${ }^{16,17}$ Despite these reports, no trials of this construct are planned for patients with BAC.

\section{Inhalational Therapy: Interferon- $\alpha$, Doxorubicin, Cisplatin, and Indomethacin}

A number of phase II trials and case reports of inhaled therapy have been described, with endpoints of tumor control and palliation of bronchorrhea. Ten patients with locally advanced BAC were treated with interferon-alpha as an inhaled aerosol with initial doses from 1 to 10 million units three times weekly and escalated to 20 million units daily. ${ }^{18}$ No symptomatic or radiographic responses were seen. Aerosolized doxorubicin has been administered to patients with primary and secondary pulmonary malignancies, including six with NSCLC and at least one with BAC. Efficacy data have not yet been reported. ${ }^{19}$

\section{Bronchorrhea: A Defining Symptom of BAC}

Bronchorrhea, defined as the production of sputum of $>100 \mathrm{ml} /$ day, is a defining symptom of BAC. Seen in about $5 \%$ of patients, bronchorrhea causes substantial discomfort. Successful treatment of BAC eliminates this condition. A variety of treatments directed at this symptom have been studied, including inhaled indomethacin, ${ }^{20,21}$ oral and inhaled corticosteroids, ${ }^{22}$ and macrolide antibiotics. ${ }^{23,24}$ Gefitinib inhibits mucin production ${ }^{25}$ and decreases bronchorrhea. ${ }^{26-29}$

\section{Response Assessment in BAC Trials}

Because of the diffuse nature of the pulmonary lesions often characteristic of BAC, some investigators have suggested that standard methods for response assessment may be inadequate for phase II trials conducted specifically in patients with BAC. Despite these concerns, the current standard of care includes patients with BAC in trials of NSCLC in general and routinely employs standard bidimensional or unidimensional response assessment. In addition, all four multicenter phase II trials completed exclusively in patients with BAC used standard criteria and reported no difficulties assessing response by both bidimensional ${ }^{4,5}$ and unidimensional ${ }^{6,30}$ methods of assessment.

\section{DISCUSSION}

\section{Consensus Recommendations}

- Because BAC represents a subtype of lung adenocarcinomas with unique clinical characteristics and molecular profile, studies specifically designed for this illness are warranted.

- The diagnosis of "pure" BAC requires a histologic specimen from the lung.

- A precise histologic classification of all NSCLC is needed.

- For clinical trials testing systemic therapies for activity in BAC, a histologic specimen and confirmation by central pathologic review are mandatory.

- For clinical trials testing systemic therapies for NSCLC in general, although histologic confirmation is favored, the use of either histology or cytology with a mandated central review is an acceptable alternative.

- Any feature of BAC in a pathologic specimen would qualify a patient for BAC-specific treatment or trials.

- If clinical features suggest a diagnosis of BAC (e.g., bronchorrhea, pneumonic consolidation, multifocal disease, visually normal bronchoscopy, etc.), but initial pathological testing is inconclusive, additional histological evaluation is warranted.

- A detailed cigarette-smoking history should be determined in all NSCLC patients. The minimum information necessary would classify people as current, former (stopped more than 1 year ago), or never smokers (fewer than 100 lifetime cigarettes).

- There is insufficient evidence to confirm or refute that the sensitivity of BAC tumors to chemotherapy is different from that of other types of NSCLC.

- Standard bidimensional (WHO, SWOG) or unidimensional (RECIST) methods of response assessment can be used in phase II trials conducted in persons with BAC. 
- Phase II trials have demonstrated that gefitinib and erlotinib have activity in BAC patients when given as initial treatment and after chemotherapy.

- In phase II trials of BAC patients receiving systemic therapies, the observed survival appears longer than in studies of NSCLC patients.

- Investigational therapy is acceptable at any point in the course of advanced BAC.

- Bronchorrhea is a defining symptom of BAC. It is best managed by effective treatment of BAC.

\section{Research Areas}

- A trial of initial gefitinib or erlotinib, then a platinumbased doublet versus an initial platinum-based doublet, and then gefitinib or erlotinib

- Exploration of the use of gefitinib and erlotinib both before and after surgery

- Trials of BAC-specific targeted therapies must include determination of molecular correlates for the target of interest

- Special areas to be addressed in clinical trials of systemic therapies in BAC:

- Central pathology review

- Response assessment

- Smoking history

- Prior and comorbid pulmonary diseases.

- Proposed or in-progress studies:

- Phase II trial of weekly bortezomib (Velcade) as first- or second-line treatment (conducted by the California Cancer Consortium)

- Phase II trial of twice-weekly bortezomib (Velcade) as first- or greater-line treatment (conducted by Millennium Pharmaceuticals)

- Phase II trial of pemetrexed (Alimta) as first- or secondline treatment (conducted by SWOG)

- Phase II trial of cetuximab (Erbitux) as first- or secondline treatment (conducted By ECOG).

- Phase II IFCT 05-04 trial: effect of an early therapeutic permutation on the tumoral control of patients receiving in the first line a specific inhibitor of tyrosin kinase of EGFR (erlotinib) or a taxane-based chemotherapy (paclitaxel + carboplatin) for the treatment of nonresectable adenocarcinoma with bronchioloalveolar features.

\section{REFERENCES}

1. World Health Organization. Pathology and genetics of tumors of the lung, pleura, thymus and heart. In Travis WD, Brambilla E, MullerHermelink H, Harris C (Eds.), World Health Organization Classification of Tumors. Lyon: IARC Press, 2004, p 38.

2. Ebright MI, Zakowski MF, Martin J, et al. Clinical pattern and pathologic stage but not histologic features predict outcome for bronchioloalveolar carcinoma. Ann Thorac Surg 2002;74:1640-1646.

3. Schiller JH, Harrington D, Belani CP, et al. Comparison of four chemotherapy regimens for advanced non-small-cell lung cancer. $N$ Eng $J$ Med 2002;346:92-98.

4. West HL, Crowley JJ, Vance RB, et al. Advanced bronchioloalveolar carcinoma: A phase II trial of paclitaxel by 96-hour infusion (SWOG 9714). Ann Oncol 2005;16:1076-1080.

5. Scagliotti GV, Smit E, Bosquee L, et al. A phase II study of paclitaxel in advanced bronchioloalveolar carcinoma (EORTC trial 08956). Lung Cancer 2005;50:91-96.

6. West HL, Franklin WA, McCoy J, et al. Gefitinib therapy in advanced bronchioloalveolar carcinoma: Southwest Oncology Group (SWOG) study S0126. J Clin Oncol 2006;24:1807-1813.

7. Franklin WA, Gumerlock PH, Crowley J. EGFR, HER2 and ERB-B pathway activation in bronchioloalveolar carcinoma (BAC): analysis of SWOG 9417 and lung SPORE tissue samples. Proc Am Soc Clin Oncol 2003;22:620.

8. Hirsch FR, Scagliotti GV, Langer CJ, Varella-Garcia M, Franklin WA. Epidermal growth factor family of receptors in preneoplasia and lung cancer: perspectives for targeted therapies. Lung Cancer 2003;41 (Suppl 1):S29-S42.

9. Franklin WA, Chansky K, Gumerlock PH, et al. Association between activation of ErbB pathway genes and survival following gefitinib treatment in advanced BAC (SWOG 0126) (Abstract). Proc ASCO 2004;7015:618.

10. Hirsch FR, Varella-Garcia M, McCoy J, et al. Increased epidermal growth factor receptor gene copy number detected by fluorescence in situ hybridization associates with increased sensitivity to gefitinib in patients with bronchioloalveolar carcinoma subtypes: A Southwest Oncology Group study. J Clin Oncol 2005;23:6838-6845.

11. Kris MG, Sandler A, Miller V, et al. Cigarette smoking history predicts sensitivity to erlotinib: results of a phase II trial in patients with bronchioloalveolar cacinoma (BAC). Proc Am Soc Clin Oncol 2004;22:631S.

12. Pao W, Miller V, Zakowski M, et al. EGF receptor gene mutations are common in lung cancers from "never smokers" and are associated with sensitivity of tumors to gefitinib and erlotinib. Proc Natl Acad Sci USA 2004;101:13306-13311.

13. Wislez M, Massiani MA, Milleron B, et al. Clinical characteristics of pneumonic-type adenocarcinoma of the lung. Chest 2003;123:1868-1877.

14. Cadranel J, Debove P, Quoix E, et al. Phase II study of gefitinib (IRESSA) administered as first time treatment in patients with nonresectable, pneumonic-type adenocarcinoma (P-ADC). In Lung Cancer. Barcelona, Spain: International Association for the Study of Lung Cancer, 2005, p S367.

15. Kubba S, Adak S, Schiller J, et al. Phase I trial of adenovirus p53 in bronchioloalveolar cell lung carcinoma (BAC) administered by bronchoalveolar lavage (BAL). Proc Am Soc Clin Oncol 2000;19:487a.

16. Salgia R, Lynch T, Skarin A, et al. Vaccination with irradiated autologous tumor cells engineered to secrete granulocyte-macrophage colonystimulating factor augments antitumor immunity in some patients with metastatic non-small-cell lung carcinoma. J Clin Oncol 2003;21:624630.

17. Nemunaitis J, Sterman D, Jablons D, et al. Granulocyte-macrophage colony-stimulating factor gene-modified autologous tumor vaccines in non-small-cell lung cancer. $J$ Natl Cancer Inst 2004;96:326-331.

18. Van Zandwijk N, Jassem E, Dubbelmann R, Braat MCP, Rumke P. Aerosol application of interferon-alpha in the treatment of bronchioloalveolar carcinoma. Eur J Cancer Clin Oncol 1990;26:738-740.

19. Sharma S, White DR, Kris M, et al. A multicenter phase I clinical and pharmacologic study of inhaled doxorubicin (Resmycin) in adults with advanced solid tumors affecting the lungs. Proc Am Soc Clin Oncol 2002:302a.

20. Homma S, Kawabata M, Kishi K, et al. Successful treatment of refractory bronchorrhea by inhaled indomethacin in two patients with bronchioloalveolar carcinoma. Chest 1999;115:1465-1468.

21. Tamaoki J, Kohri K, Isono K, Nagai A. Inhaled indomethacin in bronchorrhea in bronchioloalveolar carcinoma: role of cyclooxygenase. Chest 2000;117:1213-1214.

22. Nakajima E, Hirano T, Ikeda N, et al. K-ras mutation in sputum of primary lung cancer patients does not always reflect that of cancerous cells. Int J Onc 2001;18:105-110.

23. Desaki M, Takizawa H, Ohtoshi T, et al. Erythromycin suppresses nuclear factor-kappaB and activator protein-1 activation in human bronchial epithelial cells. Biochem Biophys Res Commun 2000;267:124-128.

24. Suga T, Sugiyama Y, Fujii T, Kitamura S. Bronchioloalveolar carcinoma with bronchorrhea treated with erythromycin. Eur Resp J 1994; 7:2249-2251.

25. Kitazaki T, Soda H, Doi S, Nakano H, Nakamura Y, Kohno S. Gefitinib inhibits MUC5AC synthesis in mucin-secreting non-small cell lung cancer cells. Lung Cancer 2005;50:19-24. 
26. Takao M, Inoue K, Watanabe F, et al. Successful treatment of persistent bronchorrhea by gefitinib in a case with recurrent bronchioloalveolar carcinoma: a case report. World J Surg Oncol 2003;1:8-11.

27. Yano S, Kanematsu T, Miki T, et al. A report of two bronchioloalveolar carcinoma cases which were rapidly improved by treatment with the epidermal growth factor receptor tyrosine kinase inhibitor ZD1839 ("Iressa"(1)). Cancer Sci 2003;94:453-458.

28. Milton D, Kris MG, Gomez J, Feinstein M. Prompt control of bronchor- rhea in patients with bronchioloalveolar carcinoma treated with gefitinib (Iressa). Support Care Cancer 2005;13:70-72.

29. Kitazaki T, Fukuda M, Soda H, Kohno S. Novel effects of gefitinib on mucin production in bronchioloalveolar carcinoma; two case reports. Lung Cancer 2005;49:125-128.

30. Sandler A, Kris MG, Miller VA, et al. Phase II trial of erlotinib in patients with bronchioloalveolar carcinoma. Lung Cancer 2005;49 (Suppl 2):S38. 